\title{
Performance Analysis of Speaker Features Extracted from High-order Fractional Domains
}

\author{
Wang Jinfang ${ }^{1}$, Wang Jinbao ${ }^{2}$ \\ ${ }^{1}$ Department of Information Engineering \\ Jilin University, Changchun, 130012 China \\ email: jinfangw@yahoo.com \\ ${ }^{2}$ Northeast Normal University, 130024 China, email: jnbwang@yahoo.com
}

\begin{abstract}
An evaluation of the feature set of the vector difference(VD) based on fractional cosine and sine transform focusing on the high-order fractional domains for text-independent speaker recognition is elucidated in this paper. The experiments have been done following the principles varying the number of the vector dimension and the power of the output parameters of fractional cosine and sine transform separately. The recognition results show that when the order of primary fractional domain is fixed to be 1 and the one of secondary fractional domain is $\mathbf{0 . 9 8}$, the correct recognition rate of the VD feature matches the one of the previous MFCC feature.
\end{abstract}

\section{INTRODUCTION}

Designing the systems performing speaker recognition task are involved with the three elemental problems: how to choose and deal with the efficacious and reliable parameters to represent the speaker individuality uniquely, how to decide the comparability measures so as to facilitate the computation and how to continuously update the referenced parameters adapting to the user whose status is keeping on changing. Among these items, the rudimental issue is to obtain the superb representatives from one's utterances such as parameter sets or patterns, etc.

In the development of speaker recognition technique, a great deal of research on the effectiveness of the various features has been done. The influential one of such efforts was made by Atal[1] who disposed of the parameters including LPC coefficients, impulsive response of vocal tract, autocorrelation function and area function of vocal tract as well as cepstrum coefficients, etc. Sambur[2] examined the formant frequencies and bandwiths of vowels, the pole locations of vowels and nasal consonants, and the pitch track and time-varying property impacting recognition performance. Subsequently, addressing the redundancy of the parameters of linear prediction, he reduced the dimensionality of linear prediction space by the orthogonal transform. Another attempt to describe the time-varying cues was made by Soong and Rosenberg[3] that instantaneous spectral information was delineated by LPC cepstrum coefficients and their trajectory in the limited time window as the transitional one was represented by orthogonal polynomial. Because the physiological difference lying in short-time speech spectrum reflects the articulation custom of a speaker in a way, the short-time spectrum-based parameters are dependable in the identification process, i.e. power spectrum, speech density and its variation. In order to augment the recognition success rate, partly due to the indefinite factors, for example, which parameters are crucial, a lot of systems have adopted the feature vector set composed of mixed parameters[4][5][8]. If the correlation between the components in the combined vectors is little, the availability of the mixed feature is obviously trustworthy because they contain the characteristics from the different aspects.

Because the abovementioned approaches are based on the common time-frequency analysis method that the speech sequence is segmented into several short intervals during which the signal is usually assumed to be stationary, the studies demonstrate this subjective presumption is deficient in treating the nonstationary parts of the utterance. Exerting the regular nonstationary signal analysis method on the interval is an alternative, but the principle we must follow is not to bring too complicated procedures. Fractional cosine transform (FRCT) and fractional sine transform(FRST)[7] as the special case of the canonical cosine and sine transform provide the expedient tool of time-frequency representation. This purpose of this paper is to produce the feature extraction scheme for speaker recognition systems aiming at high-order fractional domain that the fractional order range is confined to be in the vicinity of 1 , near frequency domain, on the time-frequency plane.

The rest of this paper is as follows. In section II, the principles of fractional cosine and sine transform are presented summarily. Section III details the proposed feature extraction method. Section IV is devoted to the related experimental procedures and results. At last, the final conclusions are given.

\section{FRACTIONAL COSINE AND SINE TRANSFORM[7]}

The fractional transform[6][7][11] of a signal on the time-frequency plane may be interpreted as rotating with the angle $\phi=(a \pi) / 2$ where $a$ denotes the destination order of fractional domain from the starting fractional domain. Before discussing fractional cosine and sine transform, the notations and operator concept should be introduced at first. Let $O^{a}$ represent fractional order operator and for the signal $g(u)$ in the $u$ domain,

$$
G^{a}(v)=\left(O^{a}[g(u)]\right)(v)
$$

where $v$ is the parameter in the $a$-th order fractional domain.

Define that fractional transform is the linear integration operation. 


$$
G^{a}(v)=\int_{-\infty}^{+\infty} K^{a}(u, v) g(u) d u
$$

where $K^{a}(u, v)$ is the kernel function of fractional transform.

So as to achieve the application convenience in this paper, the fractional order variation is confined in the interval $a \in[0,1]$ which obeys the limit property.

Concretely fractional cosine transform $G_{c}^{a}(v)$ and fractional sine transform $G_{s}{ }^{a}(v)$ possess the different transform kernel $K_{c}{ }^{a}(u, v)$ and $K_{s}^{a}(u, v)$ repectively.

$$
\begin{aligned}
& K_{c}^{a}(u, v)=A_{\phi} \mathrm{e}^{j\left(\frac{u^{2}+v^{2}}{2} \cot \phi\right)} \cos [u v(\csc \phi)] \\
& K_{s}^{a}(u, v)=A_{\phi} \mathrm{e}^{j\left(\phi-\frac{\pi}{2}\right)} \mathrm{e}^{j\left(\frac{u^{2}+v^{2}}{2} \cot \phi\right)} \sin [u v(\csc \phi)]
\end{aligned}
$$

where $A_{\phi}=\sqrt{\frac{1-j \cot \phi}{2 \pi}}$ and $\phi=(a \pi) / 2$. It is evident that substituting $K_{c}^{a}(u, v)$ and $K_{s}^{a}(u, v)$ for the kernel function of equation (2) will elicit each fractional transform. In addition, the rotation additivity is another definite property for fractional cosine and sine transform.

$$
\begin{aligned}
& G_{c}{ }^{a_{1}} G_{c}{ }^{a_{2}}=G_{c}{ }^{a_{1}+a_{2}} \\
& G_{s}{ }^{a_{1}} G_{s}{ }^{a_{2}}=G_{s}{ }^{a_{1}+a_{2}}
\end{aligned}
$$

The significant property is that fractional cosine/sine transform exclusively bears even/odd eigenfunction property. According to the deduction process of them, the following procedures may be generalized,

i) Compute coefficients $\left\{g_{m} \mid m=0,1,2,3, \cdots\right\}$ based on $g_{m}=b_{m}^{-1} \int_{-\infty}^{+\infty} g(t) H_{m}(t) \mathrm{e}^{-t^{2} / 2} d t$ and $b_{m}=\int_{-\infty}^{+\infty} H_{m}^{2}(t) \mathrm{e}^{-t^{2}} d t$ where $H_{m}(t)$ is $m$-order Hermite polynomial.

ii) $d_{m}^{c}=\lambda_{c}^{a}(m) g_{m}$ or $d_{m}^{s}=\lambda_{s}^{a}(m) g_{m}$.

iii) $G_{c}^{a}(v)=\sum_{m=0}^{+\infty} d_{m}^{c} \mathrm{e}^{-v^{2} / 2} H_{m}(v)$,

$$
G_{s}^{a}(v)=\sum_{m=0}^{+\infty} d_{m}^{s} \mathrm{e}^{-v^{2} / 2} H_{m}(v) .
$$

It is not difficult to conclude that fractional cosine transform only consists of even eigenfunctions without any odd ones and fractional sine transform is merely the weighted summation of odd eigenfunctions except even ones. As a result, the most remarkable property is that when computing the mixed time and frequency components of a signal using fractional cosine transform, the odd part of the processed signal will be deprived of and on the other hand, fractional sine transform can lead to the signal's losing the even part. Meeting the peculiarity, it is preferable for fractional cosine transform to deal with even signals and for fractional sine transform to cope with odd signals.

\section{FEATURE EXTRACTION METHOD}

The decisive procedure for speaker recognition task demands that the features extracted from an utterance sufficiently tally with the individual essence residing in the desired speaker which are inconsistent with any others' at full steam. This section presents one feature structure based on the parameters in the fractional domain depending on fractional cosine and sine transform.

\section{A. Feature definition}

The limit property of fractional transform results in the limit conditions: for $a=0$, fractional domain reduces to time domain; for $a=1$, fractional domain corresponds to frequency domain perpendicular to 0-order fractional domain; when $a$ varies in the interval $[0,1]$, fractional transform is referred to as the transform of the $a$-th order fractional domain.

Let $X\left\{x_{1}, x_{2}, \cdots, x_{i}, \cdots, x_{N}\right\}$ and $Y\left\{y_{1}, y_{2}, \cdots, y_{i}, \cdots, y_{N}\right\}$ respectively denote the $N$-point parameter sequence in primary fractional domain, $a_{1}$-th order fractional domain, and secondary fractional domain, $a_{2}$-th order fractional domain. $X$ is partitioned into $K$ segments, each of which consisting of $l$ point parameters constitutes a vector $\vec{U}_{k} \quad\left\{x_{(k-1) l+1}, x_{(k-1) l+2}, \cdots, x_{k l}\right\} \quad, \quad k \in\{1,2, \cdots, K\} \quad$ whose dimension is represented as $l$-D. The parameter sequence $X$ in primary fractional domain may be projected into $K$ vectors $\left\{\vec{U}_{1}, \vec{U}_{2}, \cdots, \vec{U}_{K}\right\}$. Likewise, the sequence $Y$ is also changed into $K$ vectors $\left\{\vec{V}_{1}, \vec{V}_{2}, \cdots, \vec{V}_{K}\right\}$ where $\vec{V}_{k}$ is $\left\{y_{(k-1) l+1}, y_{(k-1) l+2}, \cdots, y_{k l}\right\}$.

The difference of the vectors with the same subscripts between primary fractional domain and secondary fractional domain is expressed as,

$$
\vec{D}_{k}=\vec{U}_{k}-\vec{V}_{k}
$$

where vector difference(VD) $\vec{D}_{k}$ forms the differential vector sequence $\left\{\vec{D}_{1}, \vec{D}_{2}, \cdots, \vec{D}_{K}\right\}$. Considering vector difference $\vec{D}_{k}$, 2-norm is usually computed by the formula $\|\vec{D}\|_{2}=\left(\sum\left|d_{i}\right|^{2}\right)^{\frac{1}{2}}$. In order to decrease the complexity, the square of 2-norm is adopted in the feature structure. Modified 2-norm sequence may be described as $D_{2-\text { norm }}\left\{\left\|\vec{D}_{1}\right\|_{2}^{2},\left\|\vec{D}_{2}\right\|_{2}^{2}, \cdots,\left\|\vec{D}_{K}\right\|_{2}^{2}\right\}$

It is well-recognized that if the signal is processed by DCT, most of the significant information may be concentrated in just a few coefficients. Therefore, DCT plays the vital role in information compression technique. After exerting DCT on $D_{2-\text { norm }}$ sequence under the condition that the reserved $R$ coefficients should cover $99 \%$ of the energy in the sequence, the final condensed feature set $F\left\{f_{1}, f_{2}, \cdots, f_{R}\right\}$ is obtained.

\section{B. $N$-point parameter sequence}

The selection of $N$-point sequences of $X$ $\left\{x_{1}, x_{2}, \cdots, x_{i}, \cdots, x_{N}\right\}$ and $Y\left\{y_{1}, y_{2}, \cdots, y_{i}, \cdots, y_{N}\right\}$ as the crude parameters is another key step which will influence the subsequent identification task.

We assume the notations $C_{F}\left\{c_{1}, c_{2}, \cdots, c_{N}\right\}$ and $S_{F}$ $\left\{s_{1}, s_{2}, \cdots, s_{N}\right\}$ separately to represent the output sequences 
stemming from fractional cosine transform and fractional sine transform of a speech frame. $N$-point sequences should be yielded by the following principle,

$$
x_{n}=c_{n}^{j}-s_{n}^{j}
$$

where $j$ denotes the power of the output sequence of FRCT/FRST and $n$ belongs to $\{1,2, \cdots, N\}$. The case for

$Y$ is alike, but its fractional domain is altered.

In order to deepen understanding of the feature set, the final 17-dimention parameter of vector difference(VD) for the typical frame is given in the Fig.1. All subsequent experiments using VD feature set are designated as 17-dimension.

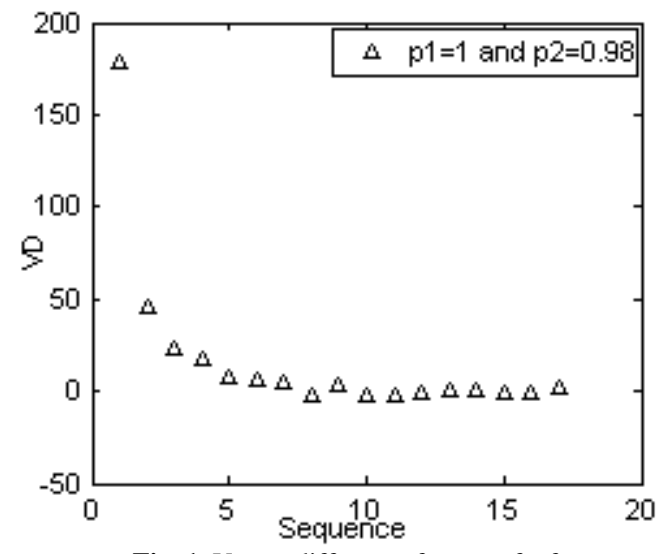

Fig. 1 Vector difference feature of a frame

\section{EXPERIMENTS AND RESULTS}

In this paper, the closed set experiments of speaker recognition have been done to identify a particular speaker as one in a finite set of reference speakers. The experiment database is the randomly chosen portion of Mandarin speech database gleaned by our laboratory members. In the database, the recorded sessions of 47 male speakers were put together to form the small database specialized in the algorithm evaluation. For one person, five talking sections each of which lasted 30s long were stored. These sessions were digitized at the sampling rate of $8 \mathrm{kHz}$ with 16 -bit sample resolution. Three sessions were used for model training and others were truncated to be $5 \mathrm{~s}$ long sessions as the testing utterances. Following the preprocessing procedures of the text-independent recognition, each training and testing utterance was segmented into several frames of $30 \mathrm{~ms}$ at a rate of $10 \mathrm{~ms}$. The silence was removed by short-time average energy method. The utterance was preemphasized with a filter coefficient of 0.97 and the frame was Hamming-windowed.

In order to evaluate the validity of our feature set, we adopt Gaussian mixture model(GMM) classifiers[9]. Every Gaussian is determined by a diagonal covariance matrix and the linear combination of eight diagonal covariance matrixes engenders one GMM. Maximum likelihood parameters modeling the speaker are estimated by the iterative expectation-maximization (EM) algorithm[10].

\section{A. Phenomenon in the whole fractional domain}

In the fractional domain whose order varies in the interval $[0,1]$, we possess the intuition that vector difference feature exhibits the different recognition performance when representing the speaker individuality. The experiments have been accomplished in the condition that the order $a_{1}$ of primary fractional domain is fixed as 1 and the one $a_{2}$ of secondary fractional domain is picked from the range $[0,1]$ with equal space. The dimension of both $\vec{U}_{k}$ and $\vec{V}_{k}$, i.e. vector dimension, is 1 , which is simply expressed as 1-D and the power $j$ of the sequence of FRCT and FRST is 3. The results in Table 1 illustrate the behavior of the different secondary fractional domains. The performance doesn't satisfy our expectation when secondary fractional domain is located in the fractional domain with the order [0.2, 0.9].

Table 1 Correct Recognition Rate for VD feature

\begin{tabular}{c|c|c|c|c}
\multicolumn{5}{c}{$(\mathrm{a} 1=1 ; j=3 ; 1-\mathrm{D})$} \\
\hline \hline ORDER $p 2$ & 0.9 & 0.8 & 0.7 & 0.6 \\
\hline Correct rate(\%) & 79.08 & 73.40 & 67.73 & 58.51 \\
\hline ORDER $P 2$ & 0.5 & 0.4 & 0.3 & 0.2 \\
\hline Correct rate(\%) & 60.64 & 57.80 & 56.38 & 57.45 \\
\hline \hline
\end{tabular}

\section{B. Effects of different $N$-point parameters}

The data in Table 1 shows the decreasing trend of recognition rate when the order of secondary fractional domain alters from 0.9 to 0.2 . Motivated by the phenomenon, we speculate that the salient VD features with the order upper than 0.9 for secondary fractional domain might presents the remarkable effects. Therefore, the following experiments have been done concentrating on this high-order fractional domain and the order of primary fractional domain is fixed as 1 uniformly.

On the other hand, according to equation (6), $N$-point parameter sequence in each fractional domain is derived from the difference between FRCT and FRST of the speech frame. It is evident that the amplitudes experiencing the transforms in the subtraction operation will be certain to influence the accuracy that the final VD feature set portrays the speaker characteristics. In the experiments, the vector dimension is fixed to be 1 alike. Table 2., 3., 4. and 5 . indicate the different values of the power $j$ of the sequence of FRCT/FRST leads to the variation of recognition performance. Fig. 2 illustrates the power increasing brings about the improvement of recognition performance approximately. Considering the computation complexity, three will be selected as the optimal power in the further experiments.

Table 2 Correct Recognition Rate for VD feature(a1=1; $j=1$; 1 -D)

\begin{tabular}{c|c|c|c|c|c}
\hline \hline ORDER $p 2$ & 0.90 & 0.91 & 0.92 & 0.93 & 0.94 \\
\hline Rate(\%) & 69.15 & 62.77 & 70.92 & 66.31 & 64.54 \\
\hline ORDER $P 2$ & 0.95 & 0.96 & 0.97 & 0.98 & 0.99 \\
\hline Rate(\%) & 73.05 & 67.02 & 73.05 & 75.53 & 73.40 \\
\hline \hline
\end{tabular}

Table 3 Correct Recognition Rate for VD feature(a1=1; $j=2$; 1 -D)

\begin{tabular}{c|c|c|c|c|c}
\hline \hline ORDER $p 2$ & 0.90 & 0.91 & 0.92 & 0.93 & 0.94 \\
\hline Rate(\%) & 79.79 & 78.37 & 79.79 & 78.37 & 81.21 \\
\hline ORDER P2 & 0.95 & 0.96 & 0.97 & 0.98 & 0.99 \\
\hline Rate(\%) & 80.85 & 82.62 & 83.33 & 84.40 & 84.04 \\
\hline \hline
\end{tabular}

Table 4 Correct Recognition Rate for VD feature(a1=1; $j=3$; 1-D)

\begin{tabular}{c|c|c|c|c|c}
\hline \hline ORDER p2 & 0.90 & 0.91 & 0.92 & 0.93 & 0.94 \\
\hline Rate(\%) & 79.08 & 79.79 & 80.50 & 80.50 & 82.98 \\
\hline ORDER P2 & 0.95 & 0.96 & 0.97 & 0.98 & 0.99 \\
\hline Rate(\%) & 82.62 & 84.75 & 85.11 & $\underline{87.94}$ & 87.23 \\
\hline \hline
\end{tabular}


Table 5 Correct Recognition Rate for VD feature(a1=1; $j=4 ; 1$-D)

\begin{tabular}{c|c|c|c|c|c}
\hline \hline ORDER $p 2$ & 0.90 & 0.91 & 0.92 & 0.93 & 0.94 \\
\hline Rate(\%) & 79.08 & 80.85 & 81.21 & 82.27 & 82.62 \\
\hline ORDER P2 & 0.95 & 0.96 & 0.97 & 0.98 & 0.99 \\
\hline Rate(\%) & 84.75 & 83.33 & 86.17 & 87.23 & 87.23 \\
\hline \hline
\end{tabular}

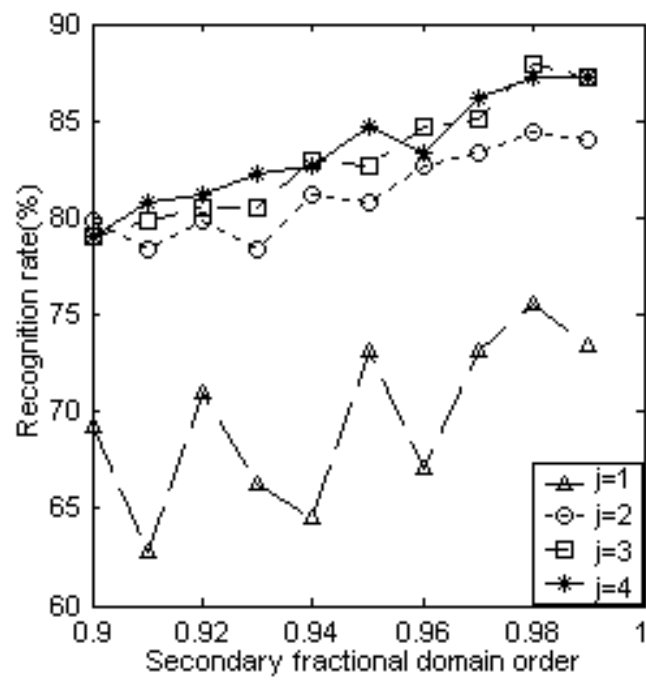

Fig. 2 Comparison of recognition performance with the different powers of the sequence of FRCT/FRST(a1=1;1-D)

\section{Effects of vector dimension variation}

The results in Table 4, 6, 7 and 8 investigating the effects of the different vector dimensions on the recognition performance are presented. Figure 2 manifests that the correct recognition rates of 1-D, 2-D, 3-D and 4-D forms the ascending curves which are entangled together. The summits of all four curves anchor at the order 0.98 of secondary fractional domain. The peak among the four summits reaches 87.94\% marking the striking achievement.

Table 6 Correct Recognition Rate for VD feature (a1=1; $j=3$; 2-D)

\begin{tabular}{c|c|c|c|c|c}
\hline \hline ORDER $p 2$ & 0.90 & 0.91 & 0.92 & 0.93 & 0.94 \\
\hline Rate(\%) & 78.72 & 79.08 & 79.08 & 81.56 & 81.56 \\
\hline ORDER P2 & 0.95 & 0.96 & 0.97 & 0.98 & 0.99 \\
\hline Rate(\%) & 82.27 & 83.33 & 86.17 & $\underline{86.17}$ & 85.11 \\
\hline \hline
\end{tabular}

Table 7 Correct Recognition Rate for VD feature(a1=1; $j=3$; 3-D)

\begin{tabular}{c|c|c|c|c|c}
\hline \hline ORDER $p 2$ & 0.90 & 0.91 & 0.92 & 0.93 & 0.94 \\
\hline Rate(\%) & 80.50 & 81.21 & 81.21 & 81.91 & 81.56 \\
\hline ORDER P2 & 0.95 & 0.96 & 0.97 & 0.98 & 0.99 \\
\hline Rate(\%) & 84.04 & 84.40 & 85.82 & $\underline{87.23}$ & 86.52 \\
\hline \hline
\end{tabular}

Table 8 Correct Recognition Rate for VD feature(a1=1; $j=3$; 4-D)

\begin{tabular}{c|c|c|c|c|c}
\hline ORDER $p 2$ & 0.90 & 0.91 & 0.92 & 0.93 & 0.94 \\
\hline Rate(\%) & 78.37 & 80.14 & 81.91 & 79.79 & 80.14 \\
\hline ORDER P2 & 0.95 & 0.96 & 0.97 & 0.98 & 0.99 \\
\hline Rate(\%) & 79.43 & 83.69 & 86.52 & $\underline{87.23}$ & 87.23 \\
\hline \hline
\end{tabular}

\section{MFCC feature function}

To establish the baseline for the succinct comparison, the recognition experiments using 12-dimension Mel frequency cepstral coefficients (MFCC) have been done. The coefficient which bore much correlation with energy was deleted. The GMM classifiers were selected to deal with the MFCC feature during training and testing procedures. The correct recognition rate is up to $86.52 \%$ which is the horizontal dashed in Fig. 3.

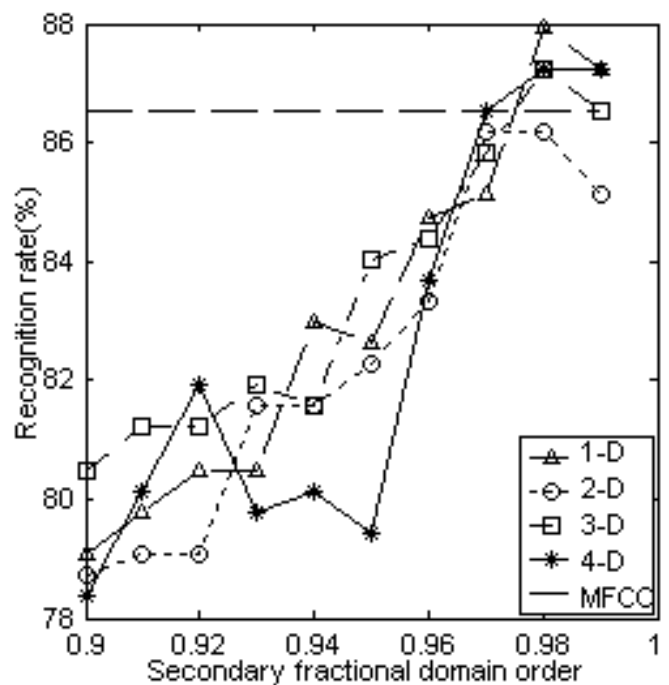

Fig. 3 Performance comparison of different vector dimensions $(\mathrm{a} 1=1 ; j=3)$

\section{CONCLUSIONS}

The speaker feature extraction method of vector difference based on high-order fractional domains has been proposed in this paper. A lot of comparison experiments demonstrate that when the order of primary fractional domain is fixed to be 1 and the power of the output sequence of fractional cosine/sine transform is selected as 3 , the recognition performance of vector difference parameters is optimal which matches the classical MFCC feature set.

\section{REFERENCES}

[1] B. S. Atal, "Effectiveness of linear prediction characteristics of the speech wave for automatic speech identification and verification”, JASA, vol. 55, no.6, pp. 1304-1320, June 1974.

[2] M. R. Sambur, "Selection of acoustic features for speaker identification”, IEEE Trans., Vol. ASSP-23, no.2, pp.176-182, April 1975.

[3] F. K. Soong and A. E. Rosenberg, "On the use of instantaneous and transitional spectral information in speaker recognition”, IEEE Trans., vol. ASSP-36, no.6, pp.871-880, June 1988.

[4] L. R. Rabiner and B.-H. Juang, Fundamentals of Speech Recognition. Englewood Cliffs, NJ: Prentice-Hall, 1993.

[5] R. J. Mannone, X. Zhang, and R. P. Ramachandran, "Robust speaker recognition: A feature-based approach,” IEEE Signal Processing Mag., vol. 13, pp. 58-71, 1996.

[6] Ç.Candan, M.A.Kutay, H.M.Ozaktas, "The discrete fractional Fourier transform”, IEEE Trans. Signal Processing, vol.48, pp.1329-1337, May 2000.

[7] S.C. Pei and M.H. Yeh, "The discrete fractional cosine and sine transform”, IEEE Trans. On Signal Processing, vol.49, pp.1198-1207, June 2001.

[8] S. Furui, "Cepstral analysis technique for automatic speaker verification,” IEEE Trans. Acoust., Speech Signal Processing, vol. ASSP-29, pp. 254-272, Apr. 1981.

[9] D. A. Reynolds and R. C. Rose, "Robust text-independent speaker identification using Gaussian mixture speaker models,” IEEE Trans. Speech Audio Processing, vol. 3, pp. 72-83, 1995.

[10]A. P. Dempster, N. M. Laird, and D. B. Rubin, "Maximum likelihood from incomplete data via the EM algorithm,” J. R. Statist. Soc., vol. 6, no. 39, pp. 1-38, 1977. 